\title{
Arbeiten in der digitalen Transformation - Chancen und Risiken für die menschengerechte Arbeitsgestaltung
}

\author{
Isabel Rothe ${ }^{1} \cdot$ Sascha Wischniewski ${ }^{2}$ Patricia Tegtmeier ${ }^{2} \cdot$ Anita Tisch $^{3}$ \\ Online publiziert: 30. August 2019 \\ (c) Der/die Autor(en) 2019
}

Schlüsselwörter Digitalisierung · Diversität von Arbeitsformen · Sicheres und gesundes Arbeiten · Partizipation . Individualisierung

\section{Einleitung: Herausforderungen für die Arbeitswissenschaft}

Die Digitalisierung durchzieht alle Bereiche der Arbeitswelt. Ein wesentlicher Treiber dieser Entwicklungen ist der Erhalt und die Steigerung der Wettbewerbsfähigkeit von Unternehmen. Dabei bleiben trotz weitreichender Bestrebungen zur Automatisierung die Beschäftigten weiterhin die zentrale Größe in der Arbeitswelt. Die mit der Digitalisierung verbundenen technologischen sowie sozialen Entwicklungen in unterschiedlichen Bereichen führen zum Teil zu grundlegenden Änderungen von Tätigkeiten und Aufgaben in allen Berufssegmenten: Es entstehen neue Arbeitsund Beschäftigungsformen ebenso wie veränderte berufliche Qualifikationsstrukturen und in der Folge neue Anforderungen an die Beschäftigten.

Diese Transformationsimpulse bieten sowohl große Chancen als auch Risiken in ihrer Wirkung auf das Gesamtsystem Arbeit. So können robotische Systeme beispielsweise repetitive und damit körperlich einseitig belastende Tätigkeiten übernehmen und dadurch die Beschäftigten physisch unterstützen oder gar entlasten. Neue Informations- und Kommunikationstechnologien eingesetzt als Assistenzsysteme bieten sich hingegen an, um kognitive Unterstützung zu leisten. Der Einsatz digitaler Technologien

Isabel Rothe

rothe.isabel@baua.bund.de

1 Bundesanstalt für Arbeitsschutz und Arbeitsmedizin (BAuA), Friedrich-Henkel-Weg 1-25, 44149 Dortmund, Deutschland

2 Fachgruppe „Human Factors, Ergonomie“, Bundesanstalt für Arbeitsschutz und Arbeitsmedizin (BAuA), Friedrich-Henkel-Weg 1-25, 44149 Dortmund, Deutschland

3 Fachgruppe „Wandel der Arbeit“, Bundesanstalt für Arbeitsschutz und Arbeitsmedizin (BAuA), Friedrich-Henkel-Weg 1-25, 44149 Dortmund, Deutschland bewirkt jedoch nicht automatisch eine belastungsoptimierte Arbeitsgestaltung, sondern kann gleichzeitig die Gefahr beinhalten, dass beim Menschen lediglich Resttätigkeiten oder einseitig belastende Tätigkeiten verbleiben. Dabei stellt der aktuelle technische Wandel keine völlig neue Herausforderung dar. Allerdings ist in der Geschwindigkeit der Transformation sowie im Ausmaß der Verbreitung neuer Technologien eine neue Qualität zu verzeichnen. Das Risiko, dass innovative Technologien und Arbeitsformen eingeführt werden, bevor Erkenntnisse zu deren Auswirkungen auf die Beschäftigten vorliegen, nimmt dadurch noch zu.

Dies stellt die Arbeitswelt und damit auch die Arbeitswissenschaft vor umfangreiche Herausforderungen. Dabei kann auf vorhandene Erkenntnisse zu menschengerechter Arbeit sowie Prinzipien ihrer Gestaltung sehr wohl zurückgegriffen werden. Gleichwohl erfordern neue Dynamiken in der technologischen und organisationalen Entwicklung erhebliche Anstrengungen, um aktuelles anwendungsorientiertes Wissen zur Verfügung zu stellen und die Gestaltungskompetenzen bei allen Beteiligten zeitgemäß weiter zu entwickeln. Hierfür sollen im Folgenden einige zentrale Eckpunkte skizziert werden.

\section{Zunehmende Diversität von Arbeitsformen und Arbeitsbedingungen in den Blick nehmen}

Im Unterschied zu der Diskussion um das „Ende der Arbeit", die unter anderem durch die Studie von Frey und Osborn (2013) ausgelöst wurde, besteht derzeit weitgehende Einigkeit darüber, dass Computer oder computergesteuerte Maschinen die wenigsten Berufe komplett ersetzen werden. Vielmehr ergeben sich umfangreiche qualitative Veränderungen auf der Ebene von Anforderungen und Tätigkeiten (Autor 2015). Grundsätzlich ist vor dem Hintergrund der 
neuen Automatisierungsoptionen davon auszugehen, dass reine Routinetätigkeiten künftig anteilig zurückgehen, während Nicht-Routinetätigkeiten immer weiter an Bedeutung gewinnen (Apt et al. 2018; Arntz et al. 2016a; Bonin et al. 2015; Dengler und Matthes 2015). Ob und wie sich diese Veränderungen konkret vollziehen werden - ob beispielsweise im Zusammenhang mit der Übernahme von Routinetätigkeiten durch neue Technologien Einfacharbeit tatsächlich obsolet wird, ob und welche neuen Tätigkeiten dadurch entstehen, wie die künftige Mensch-Maschine-Arbeitsteilung konkret aussehen könnte - wird derzeit sehr kontrovers diskutiert (Hirsch-Kreinsen 2018; Huchler und Rhein 2017; Vassiliadis 2017). Zum jetzigen Zeitpunkt ist eine unidirektionale, durch die Digitalisierung determinierte Veränderung des sozio-technischen Systems Arbeit nicht abzuleiten (Hirsch-Kreinsen 2017). Stattdessen kann sich die Nutzung digitaler Technologien in vielfältiger Weise auf die Arbeitsinhalte und -organisation auswirken (Carstensen 2015; Warning und Weber 2017).

Für die Arbeitswissenschaft stellen sich vor diesem Hintergrund zum einen grundsätzliche Gestaltungsfragen, die bereits in der Technologieentwicklung zu adressieren sind, beispielsweise hinsichtlich der Allokation zentraler Funktionen und Verantwortlichkeiten im Rahmen der Mensch-Roboter-Kooperation (Rosen und Wischniewski 2018; Adolph et al. 2018) oder der menschlichen Steuerungs- und Eingriffskompetenzen in komplexen durch Algorithmen geprägten Systemen und der Systemsicherheit. Vor allem aber muss die Arbeitswissenschaft die hohe Diversität sehr unterschiedlicher Arbeitsformen und Arbeitsbedingungen sowie deren dynamische Entwicklung in den Blick nehmen.

Für die Frage der konkreten - und nicht der grundsätzlich machbaren - Veränderungen in der Arbeitswelt ist letztlich die Umsetzung im Rahmen betrieblicher Rationalisierungsentscheidungen bedeutsam. Ob beispielsweise der Arbeitstypus der Einfacharbeit wegfällt oder in Automatisierungslücken weiter stattfindet, hängt nicht nur davon ab, ob eine technische Automatisierungslösung grundsätzlich verfügbar ist. Vielmehr sind neben den Investitionskosten viele weitere Faktoren, wie die Zuverlässigkeit der Technik, die Flexibilität der resultierenden Gesamtlösung sowie die technologische Kompetenz des Unternehmens ausschlaggebend für eine Einführung digitaler Technologien - letztlich die Frage, ob sich eine Automatisierungslösung im jeweils konkreten betrieblichen Setting insgesamt „rechnet“. Die Arbeitswissenschaft ist daher gefordert, die sich hieraus ergebende Vielfalt konkreter betrieblicher Gestaltungslösungen sowie die möglichen Auswirkungen auf die menschliche Arbeit in den Fokus zu nehmen.

\section{Gestaltungswissen für menschengerechte und wirtschaftliche Arbeit (weiter)entwickeln}

Auch wenn betriebliche Innovations- und Rationalisierungsentscheidungen wirtschaftlichen Kriterien folgen, muss dies nicht notwendigerweise im Widerspruch zu einer menschengerechten Arbeitsgestaltung stehen. Bei gut gewählten Gestaltungslösungen können beide Aspekte sogar unmittelbar zusammenwirken. So können beispielsweise strukturierte digitale Assistenzsysteme durch gezielte Arbeitsunterstützung das sozio-technische Arbeitssystem sowohl produktiver als auch sicherer gestalten (Hinrichsen und Benzioch 2019; Haase et al. 2018). Betriebliche Rationalisierungsprozesse durch neue technologische Entwicklungen bieten - auch wenn sie kostengetrieben sind aktuell und aufgrund ihrer Dynamik künftig regelmäßig die Chance, die Arbeitsgestaltung jeweils neu auszurichten und entsprechende Gestaltungslösungen oder zumindest angemessene Gestaltungskompromisse zu entwickeln. Insoweit bietet gerade die dynamische Entwicklung technologischer Innovationen Chancen für eine aktive Implementierung unter dem Aspekt der Sicherheit, Gesundheit und Wirtschaftlichkeit wünschenswerter Arbeitsformen. Hierfür zeitgemäßes Gestaltungswissen zur Verfügung zu stellen, ist eine der zentralen Herausforderungen der Arbeitswissenschaft.

Erfolgt eine Entscheidung für bestimmte Digitalisierungsvarianten im Unternehmen stattdessen überwiegend technikgetrieben, läuft sie Gefahr, den Menschen als Arbeitshandelnden aus dem Blick zu verlieren (Huchler und Rhein 2017). Im Falle einer Kooperation von Mensch und (teil-)autonomer Technik ist ein solches Vorgehen nicht nur unter arbeitswissenschaftlichen, sondern auch unter wirtschaftlichen Kriterien nicht unproblematisch. Denn für die stringente Nutzung der eingesetzten Systeme, die Ausschöpfung der Potenziale der neuen Technologien und die Erreichung der damit beabsichtigten betriebswirtschaftlichen Ziele ist die Akzeptanz der digitalen TechnologieLösungen durch die Beschäftigten ein bedeutsamer Faktor. Eine menschzentrierte Arbeitsgestaltung bildet damit auch eine entscheidende Größe für den ökonomischen Anwendungserfolg (Tegtmeier und Wischniewski 2018). Allerdings fehlen gerade für kleine und mittlere Unternehmen häufig praxisnahe Konzepte und Orientierungsmöglichkeiten für eine ebenso funktionale wie menschzentrierte Umsetzung digitaler Maßnahmen. Ohne entsprechende Strategien werden dann, etwa aus Kostengründen, Lösungen gewählt, welche sich auf alle Aspekte der Arbeitsgestaltung negativ auswirken können. Dies kann insbesondere vor dem Hintergrund begrenzter Ressourcen sowohl für die Beschäftigten als auch für die Unternehmen mitunter drastische Folgen haben (Huchler und Rhein 2017). 
Die Arbeitswissenschaft hat daher die betriebliche Umsetzungspraxis systematisch in den Blick zu nehmen um handhabbares Gestaltungswissen (weiter) zu entwickeln. Dies erfordert zum einen fundierte und repräsentative Studiendaten über den operativen Technikeinsatz vor Ort und seine konkreten Auswirkungen (Ahlers 2018). Zum anderen sollten betriebliche Umsetzungsprozesse und Gestaltungsmodelle begleitet sowie - falls in Beratungskontexten möglich - aktiv mitgestaltet und ausgewertet werden. Nur so können Erkenntnisse aus experimentellen Erhebungen im Labor in der betrieblichen Praxis validiert und Erkenntnisse über konkrete Anwendung von Technologien in der betrieblichen Praxis, deren Rahmenbedingungen und langfristige Auswirkungen ermittelt werden. Um der Vielfalt moderner Arbeitsformen gerecht zu werden, ist es dabei notwendig, ein relevantes Spektrum von Branchen und Tätigkeiten in den Blick zu nehmen.

\section{Gestaltungsbedarfe für Branchen jenseits von Industrie 4.0 erkennen}

Der Betrachtungsfokus des Einsatzes neuer digitaler Technologien lag bislang häufig auf der industriellen Produktion. Demgegenüber ist die Anwendung dieser Technologien in der Dienstleistung insbesondere im wissensintensiven Bereich weit verbreitet (Arntz et al. 2016b). Die Möglichkeiten digitaler Vernetzung, die neben höherer Flexibilität und Ressourceneinsparung neue Arten der Kundeninteraktion ermöglichen, stehen hier im Vordergrund und verändern die Arbeitsbedingungen weitreichend (Dengler und Matthes 2015; Daum 2017). Berufsgruppen und -segmente unterscheiden sich nicht nur hinsichtlich der für sie charakteristischen Tätigkeiten und entsprechender Arbeitsanforderungen, die bei der Gestaltung maßgeblich zu berücksichtigen sind. Auch ist recht unterschiedlich, wie stark sie bereits heute eine Durchdringung mit digitalen Technologien vorhanden ist und wie sehr sie sich durch die Einführung dieser in den nächsten Jahren verändern werden (Vassiliadis 2017; Arntz et al. 2016b). Dadurch ergeben sich, etwa durch ein Nebeneinander neuer und alter Prozesse oder einen sich stetig vollziehenden Wandel der eigesetzten Technologien, auch Unterschiede in und für die Gestaltung der Arbeit.

Im Bereich personenbezogener Dienstleistungsarbeit gibt es auf Grund des demografischen Wandels insbesondere im Gesundheits- und Pflegesektor vielfältige Bemühungen zum Einsatz neuer Technologien (Apt et al. 2018). Hier wird in der Digitalisierung ein wichtiger Beitrag zur Lösung aktueller Herausforderungen dieses demografischen Wandels gesehen. Der verstärkte Einsatz neuer digitaler Technologien soll dabei sowohl zu einer Effizienzsteigerung beitragen, als auch die Arbeit der Fachkräfte erleichtern und gleichzeitig die Lebensqualität der Pfle- gebedürftigen verbessern (Daum 2017; Apt et al. 2016; Hielscher et al. 2016). Daraus ergeben sich im Bereich der Dienstleistungen mit starkem Personenbezug besondere Gestaltungsherausforderungen - zum Beispiel beim Einsatz von Robotern bei personenbezogenen und sozialen Dienstleistungen - für die das entsprechende Gestaltungswissen aus industriell geprägter Tätigkeit nur sehr eingeschränkt ableitbar ist (Hielscher et al. 2016; Kehl 2018; Böhle 2016). Gerade die Betrachtung von interaktiven und wissensbasierten Dienstleistungstätigkeiten im Zusammenhang mit dem Einsatz neuer Technologien steht am Anfang einer intensiven arbeitswissenschaftlichen Forschung, die in den nächsten Jahren voranzutreiben ist (Hacker 2019).

Gleichzeitig wachsen, vermittelt durch die Digitalisierung, verschiedene Aspekte informations-, personen- und objektbezogener Arbeit auf Unternehmensebene wie auch unternehmensübergreifend immer stärker zusammen. Die Nutzung digitaler Technologien bietet in bislang unbekanntem Ausmaß eine stärkere Ausdifferenzierung von Arbeitsprozessen und die Integration unterschiedlichster auch externer Akteure mittels Plattformökonomie (HirschKreinsen 2017). Je stärker dabei eine betriebsübergreifende automatisierte Steuerung wertschöpfende Prozesse determiniert - und dies ist in Dienstleistungsbranchen besonders der Fall - desto mehr muss die Arbeitswissenschaft eine Systemperspektive einnehmen. Es müssen daher die überbetrieblichen Steuerungssysteme und Lieferketten sowie das Ineinandergreifen und Zusammenspiel ihrer einzelnen Komponenten weit mehr als bisher in den Blick genommen werden.

Im Vordergrund der Betrachtung stehen dabei der jeweils branchen- und tätigkeitsspezifische Technikeinsatz und die direkt damit verbundenen Risiken und Chancen. Vor dem Hintergrund des im Zuge der Digitalisierung verfügbaren breiten Portfolios unterschiedlicher Arbeitsmittel ist von der arbeitswissenschaftlichen Forschung dabei besondere Aufmerksamkeit auf die Chancenseite zu legen.

\section{Neue Technologien gezielt für individualisiertes Arbeiten und Teilhabe nutzen}

Bereits heute existiert ein breites Portfolio an Arbeitsassistenzsystemen, das kontextsensitiv dynamisch an die jeweilige Arbeitsperson angepasst werden kann. Dies betrifft sowohl klassisch ergonomische Fragen, beispielsweise in der digitalen Erfassung arbeitsbedingter Körperhaltungen und der Ableitung ergonomischer Potenziale (Brandl et al. 2016; Thomas et al. 2016), als auch mentale Unterstützung, zum Beispiel durch kontextsensitive Informationsbereitstellung und lernförderliche Gestaltung der Arbeit (Backhaus et al. 2018; Bornewasser et al. 2018). Insbesondere vor dem 
Hintergrund der hohen Diversität moderner Belegschaften bieten diese Formen neuer Technologie das Potenzial, sich den individuellen Voraussetzungen der Beschäftigten anzupassen. Auch ist es mit moderner Technologie mehr als bisher möglich, Beschäftigten angemessene zeitliche Flexibilität, beispielweise bei traditionell taktgebundener Tätigkeit, durch eine kontextsensitive und flexible Aufgabenallokation zur Verfügung zu stellen und dadurch etwa individuelle Belastungs- und Beanspruchungsmuster zu berücksichtigen.

Eine individualisierte Arbeitsgestaltung ermöglicht nicht nur die Berücksichtigung der Unterschiede zwischen Arbeitspersonen, sondern auch intraindividuelle Unterschiede, zum Beispiel im Tagesverlauf, oder entlang der Erwerbsbiographie. Hierdurch ergeben sich insbesondere für die Gestaltung des Zusammenspiels von Arbeit und anderen Lebensbereichen neue Möglichkeiten der Orientierung an individuellen Bedürfnissen und Anforderungen. Neue Technologien bieten zudem zusätzliche Möglichkeiten zur Teilhabe von Menschen mit gesundheitlichen Beeinträchtigungen (Apt et al. 2018). Diese können auf physischer Ebene unmittelbar kompensatorisch eingesetzt werden, beispielsweise durch die Nutzung neuartiger Sprachprozessoren, eine hybride Zusammenarbeit von Mensch und Roboter (Stern et al. 2017; Kremer et al. 2018) sowie durch den Einsatz von Exoskeletten. Gerade eine solche Teilhabe liegt nicht nur im sozialpolitischen, sondern auch im beschäftigungspolitischen Interesse. Auch können zunehmend arbeitsorganisatorische Maßnahmen wie eine Entkopplung von Arbeit von einem bestimmten Ort gezielt genutzt werden (Vassiliadis 2017) sofern resultierende Herausforderungen beispielsweise hinsichtlich neuer Anforderung an Führung Berücksichtigung finden (Weber et al. 2018).

Die Arbeitswissenschaft ist gefordert, die neuen Technologien fortlaufend zu analysieren, um zeitgemäßes Gestaltungswissen zur Verfügung stellen zu können. Die Chancen für eine individuelle Arbeitsgestaltung sind groß. Gleichzeitig können aber beispielsweise mit dem Einsatz digitaler Assistenzsysteme Risiken wie steigende Möglichkeiten zur Überwachung am Arbeitsplatz einhergehen (Hirsch-Kreinsen 2017; Backhaus 2018). Zudem bergen adaptive Assistenzsysteme und eine zunehmende digitale Vernetzung der Arbeit auch steigende Herausforderungen hinsichtlich der Systemtransparenz - sowohl auf Einzelkomponentenbasis als auch im Gesamtablauf. So muss für die Beschäftigten jederzeit offensichtlich sein, was die Technologie kann, um Bedienungsfehler ebenso wie ein Übervertrauen in die Technik zu verhindern. Solche Risiken, insbesondere auch im Kontext der Steuerungssicherheit, sind gleichzeitig in den Blick zu nehmen und (Mindest-)Standards für gute Arbeit in diesem Kontext konsequent zu formulieren. Diese sollten bereits im technologischen Entwicklungs- spätestens aber im Einführungsprozess im Unternehmen berücksichtigt werden (Backhaus 2018; Funk et al. 2019). Besonders herausfordernd hierbei sind die rasante technologische Entwicklung sowie das hohe Ausmaß der Verbreitung technologischer Anwendungen in der digitalen Transformation.

\section{Gestaltungskompetenzen bei betrieblichen Akteuren weiterentwickeln}

In Unternehmen wird häufig nicht nur ein Veränderungsprojekt nach dem anderen durchgeführt - manche technikgetrieben, manche durch die Dynamik auf der Meso- oder Makroebene initiiert - sondern auch die vermeintliche Routinearbeit vor Ort ist durch eine hohe Variabilität gekennzeichnet (Staab und Nachtwey 2016; Terhoeven et al. 2019). Dazu gehören auch die Herausforderungen bzw. Chancen und Risiken, die sich durch eine stärkere Zeit- und manchmal auch Ortsflexibilität der Arbeit ergeben, wie sie eine zunehmende Digitalisierung ermöglicht (Eurofound 2017; Messenger und Gschwind 2016). Damit wandeln sich auch die Anforderungen im Bereich der Führung durch den fortschreitenden Einsatz digitaler Technologien: Führung auf Distanz, Veränderungsmanagement oder auch eine gesundheitsgerechte Führung werden zu zentralen Führungskompetenzen, um den dynamischen Veränderungen und neuen Anforderungen an Führung gerecht zu werden (Schwarzmüller et al. 2017).

Prozesse der Arbeitsgestaltung, wie auch organisationale und technische Veränderungen, werden häufig nicht mehr in strukturierten Phasen guten Projektmanagements durchgeführt. Stattdessen ist von permanenten Wandlungsprozessen auszugehen (Köper et al. 2014). Daher muss Arbeitsgestaltung auch in operativen Alltagsprozessen stattfinden, die häufig von der Interaktion mit dem operativen Beschäftigen stark geprägt sind (Dehnborstel 2018). Vor diesem Hintergrund ist es von zentraler Bedeutung, neben der Expertise von Fachleuten, auch die Gestaltungskompetenzen der Akteure vor Ort und das Zusammenwirken aller Beteiligten mit in den Blick zu nehmen.

Weiterhin führt die Einführung immer neuer Technologien auch zu einer hohen Veränderungsfrequenz in den Arbeitsinhalten und -prozessen. Dies erfordert eine stetige Anpassung der Qualifikationen und Fähigkeiten der Beschäftigten wie auch von Führungskräften, um diese beständig auf den erforderlichen Stand zu bringen. Diskutiert wird in diesem Zusammenhang ein Wandel der Anforderungsniveaus in der Arbeit insgesamt. Erwartet werden vor allem ein Anstieg im Bereich der komplexen Tätigkeiten (Weber 2017) und damit eine Aufwertung von Tätigkeiten und Qualifikationen. Gleichzeitig ist davon auszugehen, dass auch Tätigkeiten im Helferbereich mit fortschreitender Digitalisierung nicht verschwinden, sondern einfache Tätigkeiten bleiben (Hirsch-Kreinsen 2017). Dabei nehmen auch auf niedrigem Anforderungsniveau Erwartungen an Kompeten- 
zen und Qualifikationen zu, etwa um die hierfür notwendigen Assistenzsysteme adäquat bedienen zu können. Auch müssen vor dem Hintergrund des stetigen Wandels von allen Beschäftigtengruppen immer wieder Veränderungen bewältigt und neue Anforderungen angenommen werden. Kontinuierliches Lernen und Weiterbildung werden damit in Zukunft einen immer größeren Teil der Arbeitswelt ausmachen (Bonekamp und Sure 2015; Schwahn et al. 2018).

Gute Arbeitsgestaltung in operativen Prozessen muss also unbedingt die Beschäftigten selber, deren Qualifizierung sowie deren (Selbst-)Gestaltungskompetenzen fördern. Das heißt natürlich nicht, Standards guter Arbeits- und Technikgestaltung zu vernachlässigen - im Gegenteil, hier ist zusätzliche Fachexpertise gefragt. Aber die Gestaltungskompetenzen der Beschäftigten, der Akteure vor Ort sind künftig mehr denn je von zentraler Bedeutung. Hierfür angemessene Prozesse (weiter) zu entwickeln, hinsichtlich der betrieblichen Gestaltungspraxis, der entsprechenden lernförderlichen Arbeitsgestaltung sowie der betrieblichen und überbetrieblichen Qualifizierung ist eine der wichtigsten Herausforderungen für die Arbeitswissenschaft.

\section{Zusammenfassung und Ausblick}

Die Digitalisierung der Arbeitswelt wird in ihrer Dynamik und Komplexität noch weiter zunehmen. Die mittel- und langfristigen Konsequenzen für die Gestaltung menschengerechter Arbeit lassen sich zum jetzigen Zeitpunkt nur bedingt absehen. Allerdings können bereits aufgrund der bisherigen Entwicklungen die in diesem Beitrag diskutierten Aspekte in Form der folgenden Thesen zusammengefasst werden:

- Auch wenn die Arbeitswelt sich im Zuge der digitalen Transformation grundlegend wandelt, wird uns Menschen die Arbeit nicht ausgehen. Stattdessen werden voraussichtlich weitreichende qualitative Veränderungen stattfinden und die Diversität der Arbeitsformen und Arbeitsbedingungen weiter zunehmen.

- Wie sich der künftige Technikeinsatz und der Wandel der Arbeit konkret gestalten, wird wesentlich von den betrieblichen Rationalisierungsstrategien abhängen. Hier gilt es, betriebliche Modelle zu schaffen, die menschengerechte Arbeitsgestaltung und wirtschaftliche Ziele miteinander vereinbaren.

- Die digitale Transformation bzw. vierte industrielle Revolution betrifft dabei nicht mehr vornehmlich industriell geprägten Tätigkeitsbereiche, sondern in hohem Umfang auch Dienstleistungstätigkeiten - quer durch alle Branchen - sowie neben innerbetrieblichen auch überbetriebliche Prozesse.
- Die (technischen) Möglichkeiten für eine differentielle Arbeitsgestaltung, die inter- und intraindividuelle Varianzen berücksichtigt, sind so groß wie nie zuvor. Dies eröffnet neue Chancen für die menschengerechte Arbeitsgestaltung sowie für Teilhabe.

- Die digitale Transformation führt zu einem dynamischen Wandel der Arbeitswelt, der gekennzeichnet ist durch hohe Variabilität der Arbeitsbedingungen und eine hohe Veränderungsfrequenz. Dieses stellt umfangreiche Anforderungen an die betrieblichen Akteure vor Ort, nicht zuletzt an die Beschäftigten selbst.

Die arbeitswissenschaftliche Forschung ist vor diesem Hintergrund umfassend gefordert, adäquates Wissen für eine menschzentrierte Arbeits- und Technikgestaltung in einer zunehmend digitalen Arbeitswelt weiter zu entwickeln und in den dynamischen Veränderungsprozess einfließen zu lassen. Unser Anspruch dabei ist es, die Arbeit von morgen nicht nur effizienter und produktiver zu gestalten, sondern die Chancen der digitalen Transformation auch im Sinne sicherer, gesunder und guter Arbeit zu nutzen.

Open Access Dieser Artikel wird unter der Creative Commons Namensnennung 4.0 International Lizenz (http://creativecommons.org/ licenses/by/4.0/deed.de) veröffentlicht, welche die Nutzung, Vervielfältigung, Bearbeitung, Verbreitung und Wiedergabe in jeglichem Medium und Format erlaubt, sofern Sie den/die ursprünglichen Autor(en) und die Quelle ordnungsgemäß nennen, einen Link zur Creative Commons Lizenz beifügen und angeben, ob Änderungen vorgenommen wurden.

\section{Literatur}

Adolph L, Kirchhoff B, Geilen JH (2018) Sicherheit und Gesundheit in der digitalen Arbeitswelt. In: Maier G, Engels G, Steffen E (Hrsg) Handbuch Gestaltung digitaler und vernetzter Arbeitswelten. Springer Reference Psychologie. Springer, Berlin, Heidelberg, S 1-14

Ahlers E (2018) Die Digitalisierung der Arbeit. Verbreitung und Einschätzung aus Sicht der Betriebsräte. WSI-Report 40

Apt W, Bovenschulte M, Hartmann EA, Wischmann S (2016) Foresight-Studie „Digitale Arbeitswelt“. Forschungsbericht 463 für das Bundesministerium für Arbeit und Soziales. Institut für Innovation und Technik, Berlin

Apt W, Bovenschulte M, Priesack K, Weiß C, Hartmann EA (2018) Einsatz von digitalen Assistenzsystemen im Betrieb. Forschungsbericht 502. Institut für Innovation und Technik, Berlin

Arntz M, Gregory T, Jansen S, Zierahn U (2016a) Tätigkeitswandel und Weiterbildungsbedarf in der digitalen Transformation. ZEW, Mannheim

Arntz M, Gregory T, Lehmer F, Matthes B, Zierahn U (2016b) Arbeitswelt 4.0 - Stand der Digitalisierung in Deutschland. Dienstleister haben die Nase vorn. IAB Kurzbericht 22/2016. IAB, Nürnberg

Autor DH (2015) Why are there still so many jobs? The history and future of workplace automation. J Econ Perspect 29(3):3-30

Backhaus N (2018) Review zur Wirkung elektronischer Überwachung am Arbeitsplatz und Gestaltung kontextsensitiver Assistenzsysteme (baua: Bericht). Bundesanstalt für Arbeitsschutz und Arbeitsmedizin, Dortmund, Berlin, Dresden

Backhaus N, Knittel M, Weisner K, Benter M, Wischniewski S, Jaitner T, Deuse J (2018) Förderung gesunder Arbeit durch kon- 
textsensitive Assistenzsysteme in der industriellen Fertigung und Montage. In: GfA (Hrsg) ARBEIT(s).WISSEN.SCHAF(f)T Grundlage für Management \& Kompetenzentwicklung. GfA, Dortmund

Böhle F (2016) Das Programm „Humanisierung der Arbeit“ und Interaktionsarbeit. In: ver. di-Bereich Innovation und Gute Arbeit (Hrsg) Arbeiten mit Menschen - Interaktionsarbeit humanisieren. Band 1: Gestaltungskonzepte und Forschungsbedarf. Bund, Frankfurt, S 59-67

Bonekamp L, Sure M (2015) Consequences of Industry 4.0 on human labour and work organisation. J Bus Media Psychol 6(1):33-40

Bonin H, Gregory T, Zierahn U (2015) Übertragung der Studie von Frey/Osborne (2013) auf Deutschland. Forschungsbericht 455. ZEW, Mannheim

Bornewasser M, Bläsing D, Hinrichsen S (2018) Informatorische Assistenzsysteme in der manuellen Montage: Ein nützliches Werkzeug zur Reduktion mentaler Beanspruchung? Z Arbeitswiss 72:264-275

Brandl C, Bonin D, Mertens A, Wischniewski S, Schlick CM (2016) Digitalisierungsansätze ergonomischer Analysen und Interventionen am Beispiel der markerlosen Erfassung von Körperhaltungen bei Arbeitstätigkeiten in der Produktion. Z Arbeitswiss 70:89-98

Carstensen T (2015) Neue Anforderungen und Belastungen durch digitale und mobile Technologien. WSI Mitt 2015(3):187-193

Daum M (2017) Digitalisierung und Technisierung der Pflege in Deutschland. Aktuelle Trends und ihre Folgewirkungen auf Arbeitsorganisation, Beschäftigung und Qualifizierung. DAAStiftung Bildung und Beruf, Hamburg

Dehnbostel P (2018) Lern- und kompetenzförderliche Arbeitsgestaltung in der digitalisierten Arbeitswelt. Arbeit 27(4):269-294

Dengler K, Matthes B (2015) Folgen der Digitalisierung für die Arbeitswelt. Substituierbarkeitspotenziale von Berufen in Deutschland. IAB Forschungsbericht 11/2015

Eurofound and the International Labour Office (2017) Working anytime, anywhere: The effects on the world of work. EUR-OP, ILO, Luxembourg, Geneva

Frey CB, Osborn MA (2013) The future of employment: How susceptible are jobs to computerization? Working paper. Oxford Martin Programme on Technology and Employment, Oxford

Funk M, Backhaus N, Terhoeven J, Wischniewski S (2019) Menschzentrierte Gestaltung digitaler Arbeitsassistenz: Herausforderungen hinsichtlich Überwachung und Datenschutz kontextsensitiver Systeme. In: GfA (Hrsg) Arbeit interdisziplinär analysieren - bewerten - gestalten. GfA, Dortmund

Haase V, Mzyk M, Strecker M, Wondsak I (2018) Agile Softwareentwicklung in einer Industrie 4.0. In: Bauer W, Marrenbach D (Hrsg) MyCPS - Migrationsunterstützung für die Umsetzung menschzentrierter Cyber-Physical Systems. Abschlussbericht. IAO, Stuttgart, S 59-70

Hacker W (2019) Menschengerechtes Arbeiten in der digitalisierten Welt. Vdf Hochschulverlag, Zürich

Hielscher V, Nock L, Kirchen-Peters S (2016) Technikvermittlung als Anforderung in der Dienstleistungsinteraktion. Empirische Befunde zum Technikeinsatz in der Altenpflege. Arbeit 25(1-2):3-19

Hinrichsen S, Bendzioch S (2019) How digital assistance systems improve work productivity in assembly. In: Nunes I (Hrsg) Advances in human factors and systems interaction, Bd. 781. Springer, Cham, S 332-342

Hirsch-Kreinsen H (2017) Industrie und Arbeit 4.0: Entwicklungstrends und Gestaltungsperspektiven. In: Vassiliadis M (Hrsg) Digitalisierung und Industrie 4.0. Technik allein reicht nicht. BWH, Hannover, S 115-133
Hirsch-Kreinsen H (2018) Einleitung: Digitalisierung industrieller Arbeit. In: Hirsch-Kreinsen H, Ittermann P, Niehaus J (Hrsg) Digitalisierung industrieller Arbeit, die Vision Industrie 4.0 und ihre sozialen Herausforderungen, 2. Aufl. Nomos, Baden-Baden, S $13-32$

Huchler N, Rhein P (2017) Arbeitshandeln und der digitale Wandel von kleinen und mittleren Unternehmen. Die Rolle des Menschen und die Grenzen der Formalisierung 4.0. Arbeit 26(3-4):287-314

Kehl C (2018) Entgrenzung zwischen Mensch und Maschine, oder: können Roboter zu guter Pflege beitragen? Polit Zeitgesch 68(68):22-28

Köper B, Seiler K, Beerheide E (2014) Restrukturierung und Gesundheit. Z Arbeitswiss 4:243-253

Kremer D, Hermann S, Henkel C (2018) Mensch-Roboter-Kollaboration für Schwerbehinderte als Beitrag zur Inklusion in der Arbeitswelt. innteract2018 (3), Beitrag 284

Messenger JC, Gschwind L (2016) Three generations of telework: New ICT and the (r)evolution from home office to virtual office. New Technol Work Employ 31(3):195-208

Rosen PH, Wischniewski S (2018) Task design in human-robot-interaction scenarios - Challenges from a human factors perspective. In: Nunes I (Hrsg) Advances in human factors and systems interaction. Springer, Cham, S 71-82

Schwahn F, Mai C-M, Braig M (2018) Arbeitsmarkt im Wandel - Wirtschaftsstrukturen, Erwerbsformen und Digitalisierung. Wirtsch Stat 2018(03):24-39

Schwarzmüller T, Brosi P, Welpe IM (2017) Führung 4.0 - Wie die Digitalisierung Führung verändert. In: Hildebrandt A, LandhäuBer W (Hrsg) CSR und Digitalisierung. Management-Reihe Corporate Social Responsibility. Springer Gabler, Berlin, Heidelberg, S 617-662

Staab P, Nachtwey O (2016) Die Digitalisierung der Dienstleistungsarbeit. Polit Zeitgesch 66(18-19):24-31

Stern SE, Chobany CM, Beam AA, Hoover BN, Hull TT, Linsenbigler M, Makdad-Light C, Rubright CN (2017) Use of speech generating devices can improve perception of qualifications for skilled, verbal, and interactive jobs. Work 56:199-211

Tegtmeier P, Wischniewski S (2018) Usability-Erwartungen an die Einführung menschzentrierter cyber-physischer System in der Produktion 4.0. In: GfA (Hrsg) ARBEIT(s).WISSEN.SCHAF(f)T - Grundlage für Management \& Kompetenzentwicklung. GfA, Dortmund

Terhoeven J, Mühlenbrock I, Mehler L, Ribbat M, Tisch A, Wischniewski S (2019) Lernförderlichkeit im Kontext einer menschengerechten Arbeitssystemgestaltung. In: GfA (Hrsg) Arbeit interdisziplinär analysieren - bewerten - gestalten. GfA, Dortmund

Thomas C, Stankiewicz L, Grötsch A, Wischniewski S, Deuse J, Kuhlenkötter B (2016) Intuitive work assistance by reciprocal humanrobot interaction in the subject area of direct human-robot collaboration. Procedia CIRP 44:275-280

Vassiliadis M (2017) Industrie 4.0 braucht soziale Innovationen. In: Vassiliadis M (Hrsg) Digitalisierung und Industrie 4.0. Technik allein reicht nicht. BWH, Hannover, S 13-35

Warning A, Weber E (2017) Wirtschaft 4.0. Digitalisierung verändert die betriebliche Personalpolitik. Institut für Arbeitsmarkt- und Berufsforschung der Bundesagentur für Arbeit, Nürnberg

Weber E (2017) Wirtschaft 4.0: Beschäftigung, Arbeitsmarkt, Qualifikation. In: Vassiliadis M (Hrsg) Digitalisierung und Industrie 4.0. Technik allein reicht nicht. BWH GmbH, Hannover, S 95-113

Weber C, Thomson B, Pundt F (2018) Die Notwendigkeit von Führung in einer digitalisierten Arbeitswelt - eine Netnografie. Bundesanstalt für Arbeitsschutz und Arbeitsmedizin, Dortmund 